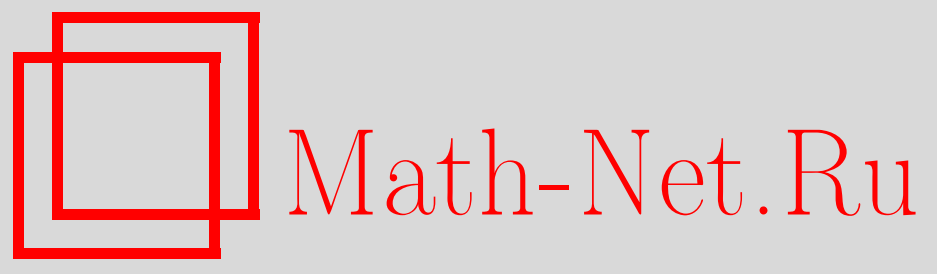

E. K. Башкиров, Е. Г. Мангулова, Перепутывание атомов, индуцированное двухмодовым тепловым шумом, при наличии диполь-дипольного взаимодействия и атомной когерентности, Вестн. Сам. гос. техн. унта. Сер. Физ.-мат. науки, 2013, выпуск 2(), 177-184

DOI: https://doi.org/10.14498/vsgtu1160

Использование Общероссийского математического портала Math-Net.Ru подразумевает, что вы прочитали и согласны с пользовательским соглашением

http://www . mathnet.ru/rus/agreement

Параметры загрузки:

IP : 54.197 .217 .227

26 апреля 2023 г., 02:19:58

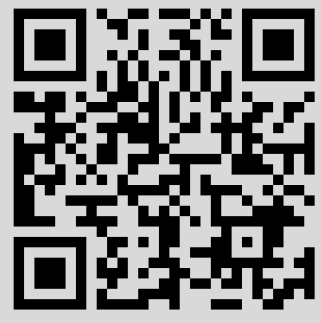


УДК 535.14

\title{
ПЕРЕПУТЫВАНИЕ АТОМОВ, ИНДУЦИРОВАННОЕ ДВУХМОДОВЫМ ТЕПЛОВЫМ ШУМОМ, ПРИ НАЛИЧИИ ДИПОЛЬ-ДИПОЛЬНОГО ВЗАИМОДЕЙСТВИЯ И АТОМНОЙ КОГЕРЕНТНОСТИ
}

\author{
Е. К. Башкиров, Е. Г. Мангулова \\ Самарский государственный университет, \\ Россия, 443011, Самара, ул. Академика Павлова, 1. \\ E-mails: bash@samsu.ru, mangulova-eg@mail.ru
}

\begin{abstract}
Рассмотрено перепутывание двух двухуровневых атомов с невырожденными двухботонными переходами, взаимодействующих с тепловым двухмодовым полем в идеальном резонаторе. Показана возможность значительного увеличения степени атомного перепутывания при наличии атомной когерентности и прямого диполь-дипольного взаимодействия атомов.
\end{abstract}

Ключевые слова: атомное перепутывание, тепловое двухмодовое поле, дипольдипольное взаимодействие, атомная когерентностъ.

Введение. Проблема описания динамики кубитов, взаимодействующих с электромагнитными полями, является одной из наиболее актуальных проблем современной физики квантовых вычислений. Такие системы активно изучаются как с теоретической, так и с экспериментальной точек зрения в целом ряде экспериментальных установок: одноатомных мазерах и лазерах, ионах в резонаторах и магнитных ловушках, сверхпроводящих системах на джозефсоновских переходах, квантовых точках и других искусственных микрообъектах [1]. Такие исследования позволили создать прообразы квантовых компьютеров, работающих пока на отдельных кубитах. Дальнейший прогресс в данной области требует исследования новых физических устройств, которые можно использовать в качестве логических элементов квантовых компьютеров, для выбора оптимальных режимов работы таких систем, в том числе наиболее эффективных схем генерации и контроля перепутывания состояний кубитов, а также особенностей их релаксации. Для приложений в физике квантовых вычислений нужны максимально перепутанные чистые состояния с достаточно большим временем жизни, однако в реальных условиях квантовые системы всегда взаимодействуют с окружением. Такое взаимодействие обычно приводит к декогерентности, так что исследуемая система эволюционирует в смешанное неперепутанное состояние, которое оказывается непригодным для целей квантовых вычислений. Поэтому с практической точки зрения основная задача при получении и использовании атомных перепутанных состояний заключается в том, чтобы минимизировать влияние шума. Недавно в целом ряде работ была высказана идея о том, что в некоторых случаях диссипация и шум могут, напротив, являться источником перепутывания. В частности, была показана возможность генерации перепутывания атомных систем в резонаторах, индуцированных тепловым шумом. В

Евгений Константинович Башкиров (д.ф.-м.н., проф.), профессор, каф. общей и теоретической физики. Екатерина Геннадъевна Мангулова, студент, каф. общей и теоретической физики. 
работе [2] было показано, что одномодовый тепловой шум может индуцировать атом-атомное перепутывание в системе двух двухуровневых атомов в идеальном резонаторе. Позднее аналогичное поведение было обнаружено и для атомов с многофотонными переходами [3,4]. При этом было показано, что при двухфотонном взаимодействии атомов с тепловым полем степень перепутывания атомных состояний может значительно превосходить соответствующую величину для однофотонного взаимодействия. Позднее в целом ряде работ исследовались особенности генерации атомного перепутывания в различных обобщениях двухатомной модели Тависа-Каммингса для резонаторного поля в тепловом состоянии (см. ссылки в работе [5]).

Недавно на примере системы двух атомов, взаимодействующих как с одномодовым [9], так и с двухмодовым тепловым полем [10], было показано,что степень перепутывания атомов сильно зависит от их начального состояния. Показано, что при наличии атомной когерентности степень атомного перепутывания может заметно возрастать, а также, что степенью атомного перепутывания можно управлять, изменяя относительные фазы и амплитуды поляризованных атомов. При этом авторы не учли диполь-дипольного взаимодействия атомов. Вместе с тем в ряде работ показано, что диполь-дипольное взаимодействие может существенно увеличить степень атомного перепутывания [6-8].

В нашей работе [11] было рассмотрено влияние атомной когерентности на степень атомного перепутывания в невырожденной двухмодовой модели Тависа-Каммингса при наличии диполь-дипольного взаимодействия атомов в случае высоких степеней теплового шума (высокой температуры резонатора). При этом было показано, что при наличии диполь-дипольного взаимодействия и атомной когерентности возможна генерации высокой степени перепутывания атомов, на порядок превосходящей степень перепутывания без учета указанных механизмов. В настоящей работе мы исследуем влияние диполь-дипольного взаимодействия и атомной когерентности на атомное перепутывание в случае низких температур резонатора. Рассмотрение такого случая представляет также интерес и в связи с тем, что в экспериментах с одноатомными мазерами и лазерами резонаторы охлаждаются до температур порядка 0,5 K. В этом случае среднее число число тепловых фотонов составляет менее 0,05 .

1. Модель и её точное решение. Рассмотрим два идентичных двухуровневых атома, резонансно взаимодействующих с двухмодовым квантовым электромагнитным полем в идеальном резонаторе посредством невырожденных двухфотонных переходов при наличии прямого диполь-дипольного взаимодействия между атомами. В представлении взаимодействия гамильтониан такой модели можно представить в виде

$$
H=\hbar g \sum_{i=1}^{2}\left(a_{1}^{+} a_{2}^{+} R_{i}^{-}+R_{i}^{+} a_{1} a_{2}\right)+\hbar \Omega\left(R_{1}^{+} R_{2}^{-}+R_{2}^{+} R_{1}^{-}\right)
$$

где $a_{j}^{+}$и $a_{j}$ - операторы рождения и уничтожения фотонов $j$-той резонаторной моды $(j=1,2), R_{i}^{+}$и $R_{i}^{-}$- повышающий и понижающий оператор в $i$-том атоме $(i=1,2), g$ - константа невырожденного двухфотонного взаимодействия атомов с полем и $\Omega$ - константа прямого диполь-дипольного взаимодействия атомов. 
Обозначим через $|+\rangle$ и $|-\rangle$ возбужденное и основное состояния двухуровневого атома. Тогда двухатомная волновая функция может быть представлена в виде комбинации волновых векторов вида $|\alpha, \beta\rangle=|\alpha\rangle|\beta\rangle$, где $\alpha, \beta=+,-$. Атом-полевая система в идеальном резонаторе обладает унитарной динамикой, которая в представлении взаимодействия описывается оператором эволюции вида $U(t)=\exp (-\imath H t / \hbar)$. Если система, включающая атомы и поле, находится в начальный момент времени в чистом состоянии, то ее вектор состояния в любой момент времени в представлении взаимодействия может быть представлен в виде $|\Psi(t)\rangle=U(t)|\Psi\rangle(0)$. В двухатомном базисе $|+,+\rangle$, $|+,-\rangle,|-,+\rangle,|-,-\rangle$ оператор эволюции $U(t)$ для модели с гамильтонианом (1) может быть записан как

$$
U(t)=\left(\begin{array}{llll}
U_{11} & U_{12} & U_{13} & U_{14} \\
U_{21} & U_{22} & U_{23} & U_{24} \\
U_{31} & U_{32} & U_{33} & U_{34} \\
U_{41} & U_{42} & U_{43} & U_{44}
\end{array}\right)
$$

Здесь

$$
\begin{aligned}
& U_{11}=1+ 2 a_{1} a_{2} \frac{A}{\lambda} a_{1}^{+} a_{2}^{+}, \quad U_{14}=2 a_{1} a_{2} \frac{A}{\lambda} a_{1} a_{2}, \quad U_{41}=2 a_{1}^{+} a_{2}^{+} \frac{A}{\lambda} a^{+} a_{1} a_{2}, \\
& U_{44}=1+ 2 a_{1}^{+} a_{2}^{+} \frac{A}{\lambda} a_{1} a_{2}, \quad U_{12}=U_{13}=a_{1} a_{2} \frac{B}{\theta}, \quad U_{21}=U_{31}=\frac{B}{\theta} a_{1}^{+} a_{2}^{+}, \\
& U_{24}=U_{34}=\frac{B}{\theta} a_{1} a_{2}, \quad U_{42}=U_{43}=a_{1}^{+} a_{2}^{+} \frac{B}{\theta}, \\
& U_{22}=U_{33}= \frac{\exp \left[-\imath \frac{g}{2}(\alpha+\theta) t\right]}{4 \theta} \times \\
& \times\left\{[1-\exp (\imath g \theta t)] \alpha+2 \theta \exp \left(\imath \frac{g}{2}(3 \alpha+\theta) t\right)+\theta[1+\exp (\imath g \theta t)]\right\}, \\
& U_{23}=U_{32}= \frac{\exp \left[-\imath \frac{g}{2}(\alpha+\theta) t\right]}{4 \theta} \times \\
& \times\left\{[1-\exp (\imath g \theta t)] \alpha-2 \theta \exp \left(\imath \frac{g}{2}(3 \alpha+\theta) t\right)+\theta[1+\exp (\imath g \theta t)]\right\},
\end{aligned}
$$

где

$$
\begin{gathered}
A=\exp \left[-\imath \frac{g \alpha}{2} t\right]\left\{\cos \left(\frac{g \theta}{2} t\right)+\imath \frac{\alpha}{\theta} \sin \left(\frac{g \theta}{2} t\right)\right\}-1 \\
B=\exp \left[-\imath \frac{g}{2}(\alpha+\theta) t\right][1-\exp (\imath g \theta t)]
\end{gathered}
$$

и $\alpha=\Omega / g, \lambda=2\left(a_{1} a_{2} a_{1}^{+} a_{2}^{+}+a_{1}^{+} a_{2}^{+} a_{1} a_{2}\right), \theta=\sqrt{8\left(a_{1} a_{2} a_{1}^{+} a_{2}^{+}+a_{1}^{+} a_{2}^{+} a_{1} a_{2}\right)+\alpha^{2}}$.

Пусть в начальный момент времени резонаторное поле находится в двухмодовом тепловом состоянии

$$
\rho_{F}(0)=\sum_{n_{1}} \sum_{n_{2}} p_{1}\left(n_{1}\right) p_{2}\left(n_{2}\right)\left|n_{1}, n_{2}\right\rangle\left\langle n_{1}, n_{2}\right|,
$$


где $p_{i}\left(n_{i}\right)=\bar{n}_{i}^{n_{i}} /\left(1+\bar{n}_{i}\right)^{n_{i}}$ и $\bar{n}_{i}$-среднее число тепловых фотонов в $i$-той моде, а атомы в когерентных атомных состояниях вида

$$
\left|\Psi_{1}(0)\right\rangle=\cos \theta_{1}|+\rangle+e^{\imath \varphi_{1}} \sin \theta_{1}|-\rangle, \quad\left|\Psi_{2}(0)\right\rangle=\cos \theta_{2}|+\rangle+e^{\imath \varphi_{2}} \sin \theta_{2}|-\rangle .
$$

Здесь $\theta_{1}$ и $\theta_{2}$ обозначают амплитуды поляризованных атомов и $\varphi_{1}$ и $\varphi_{2}-$ относительные фазы состояний двух атомов. Начальная атомная матрица плотности атомов можем быть тогда записана в виде

$$
\rho_{A}(0)=\left(\begin{array}{llll}
\rho_{11}(0) & \rho_{12}(0) & \rho_{13}(0) & \rho_{14}(0) \\
\rho_{12}^{*}(0) & \rho_{22}(0) & \rho_{23}(0) & \rho_{24}(0) \\
\rho_{13}^{*}(0) & \rho_{23}^{*}(0) & \rho_{33}(0) & \rho_{34}(0) \\
\rho_{14}^{*}(0) & \rho_{24}^{*}(0) & \rho_{34}^{*}(0) & \rho_{44}(0)
\end{array}\right)
$$

где

$$
\begin{array}{ll}
\rho_{11}(0)=\cos ^{2} \theta_{1} \cos ^{2} \theta_{2}, & \rho_{12}(0)=\cos ^{2} \theta_{1} \cos \theta_{2} \sin \theta_{2} e^{-\imath \varphi_{2}}, \\
\rho_{13}(0)=\cos \theta_{1} \sin \theta_{1} \cos ^{2} \theta_{2} e^{-\imath \varphi_{1}}, & \rho_{14}(0)=\cos \theta_{1} \sin \theta_{1} \cos \theta_{2} \sin \theta_{2} e^{-\imath\left(\varphi_{1}+\varphi_{2}\right)}, \\
\rho_{22}(0)=\cos ^{2} \theta_{1} \sin ^{2} \theta_{2}, & \rho_{23}(0)=\cos \theta_{1} \sin \theta_{1} \cos \theta_{2} \sin \theta_{2} e^{-\imath\left(\varphi_{1}-\varphi_{2}\right)}, \\
\rho_{33}(0)=\sin ^{2} \theta_{1} \cos ^{2} \theta_{2}, & \rho_{24}(0)=\cos \theta_{1} \sin \theta_{1} \sin ^{2} \theta_{2} e^{-\imath \varphi_{1}}, \\
\rho_{34}(0)=\sin ^{2} \theta_{1} \cos \theta_{2} \sin \theta_{2} e^{-\imath \varphi_{2}}, & \rho_{44}(0)=1-\rho_{11}(0)-\rho_{22}(0)-\rho_{33}(0) .
\end{array}
$$

Для вычисления критерия перепутанности атомов необходимо найти редуцированную атомную матрицу плотности

$$
\rho_{A}(t)=\operatorname{Tr}_{F} U(t) \rho_{F}(0) \otimes \rho_{A}(0) U^{+}(t)
$$

В результате с использованием явного вида оператора эволюции (2) для атомной матрицы плотности получаем

$$
\rho_{A}(t)=\left(\begin{array}{cccc}
\rho_{11} & \rho_{12} & \rho_{13} & \rho_{14} \\
\rho_{12}^{*} & \rho_{22} & \rho_{23} & \rho_{24} \\
\rho_{13}^{*} & \rho_{23}^{*} & \rho_{33} & \rho_{34} \\
\rho_{14}^{*} & \rho_{24}^{*} & \rho_{34}^{*} & \rho_{44}
\end{array}\right)
$$

Явный вид матричных элементов в (3) не приведен здесь из-за их слишком громоздкого вида.

2. Обсуждение результатов. Для определения степени атом-атомного перепутывания будем использовать параметр Переса Хородецких [1], который определяется как $\varepsilon=-2 \sum_{i} \mu_{i}^{-}$, где $\mu_{i}^{-}$- отрицательные собственные значения частично транспонированной по переменным одного кубита (атома) матрицы (3). Для неперепутанных состояний $\varepsilon=0$. Для перепутанных состояний $0<\varepsilon \leqslant 1$. Максимальной степени перепутывания соответствует значение $\varepsilon=1$.

Результаты численного моделирования временной зависимости параметра перепутывания для различных значений параметров модели представлено на рис. 1 и 2. На рис. 1, а, б показано временное поведение перепутывания для двухмодового теплового моля с малым средним числом фотонов в модах $\bar{n}_{1}=\bar{n}_{2}=0,01$ без учета диполь-дипольного взаимодействия атомов в отсутствии (рис. 1, а) и при наличии атомной когерентности (рис. 1, б, в). На рис. 1 , 

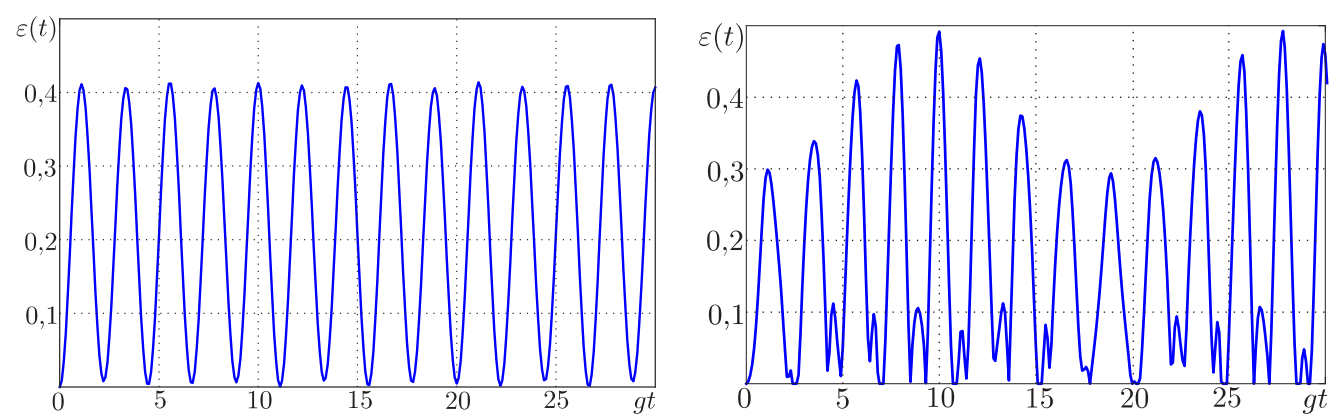

a
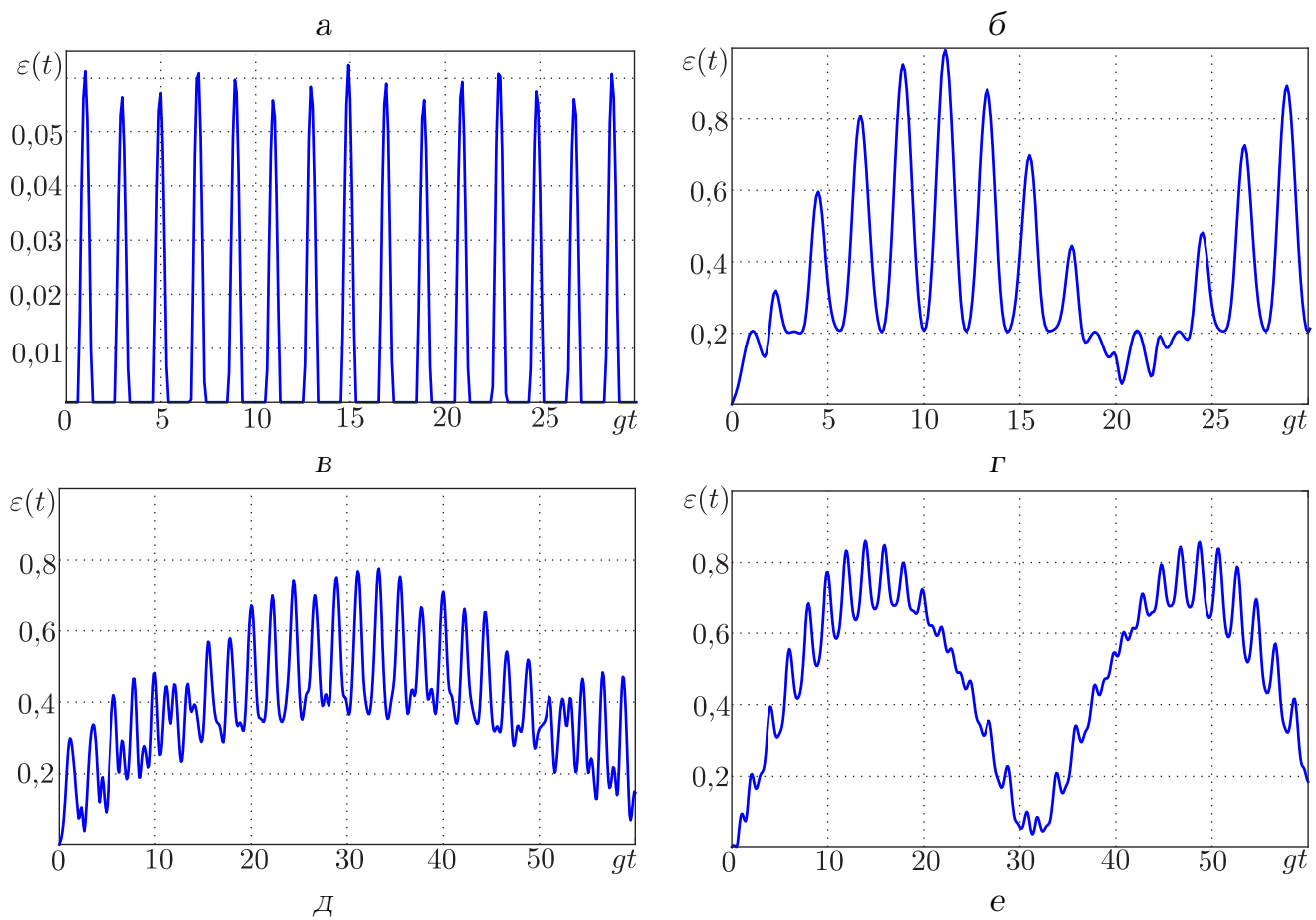

Рис. 1. Временная зависимость параметра перепутывания для параметров модели: а) $\alpha=$ $=0, \theta_{1}=\pi / 2, \theta_{2}=0$; б) $\alpha=0, \theta_{1}=\pi / 4, \theta_{2}=\pi / 4$; в) $\left.\alpha=0, \theta_{1}=\pi / 4, \theta_{2}=-\pi / 4, \Gamma\right) \alpha=0,1, \theta_{1}=$ $=\pi / 2, \theta_{2}=0$, д) $\alpha=0,1, \theta_{1}=\pi / 4, \theta_{2}=\pi / 4$, e) $\alpha=0,1, \theta_{1}=\pi / 4, \theta_{2}=-\pi / 4$; фазы поляризованных атомов $\varphi_{1}=\varphi_{2}=0$ и среднее число тепловых фотонов в модах $\bar{n}_{1}=\bar{n}_{2}=0,01$ 

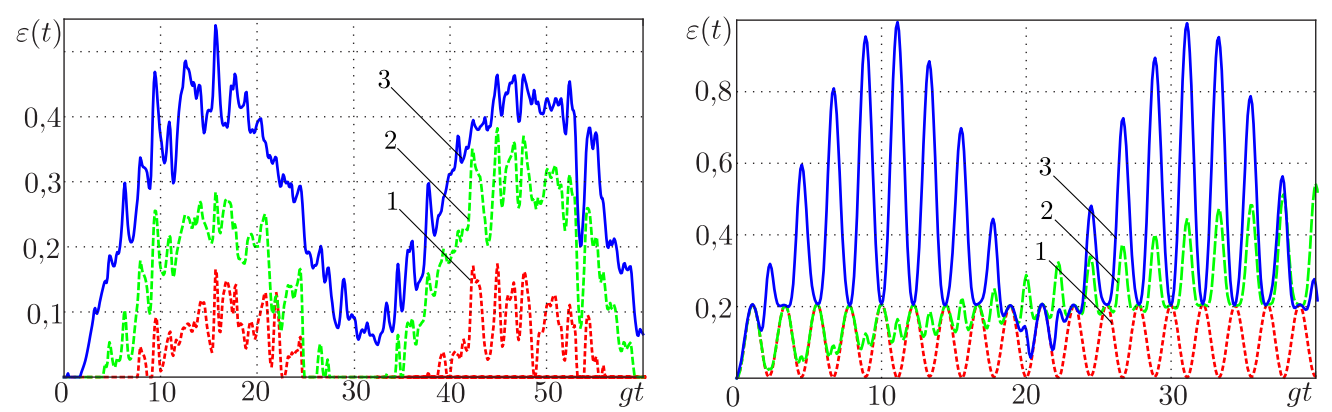

a
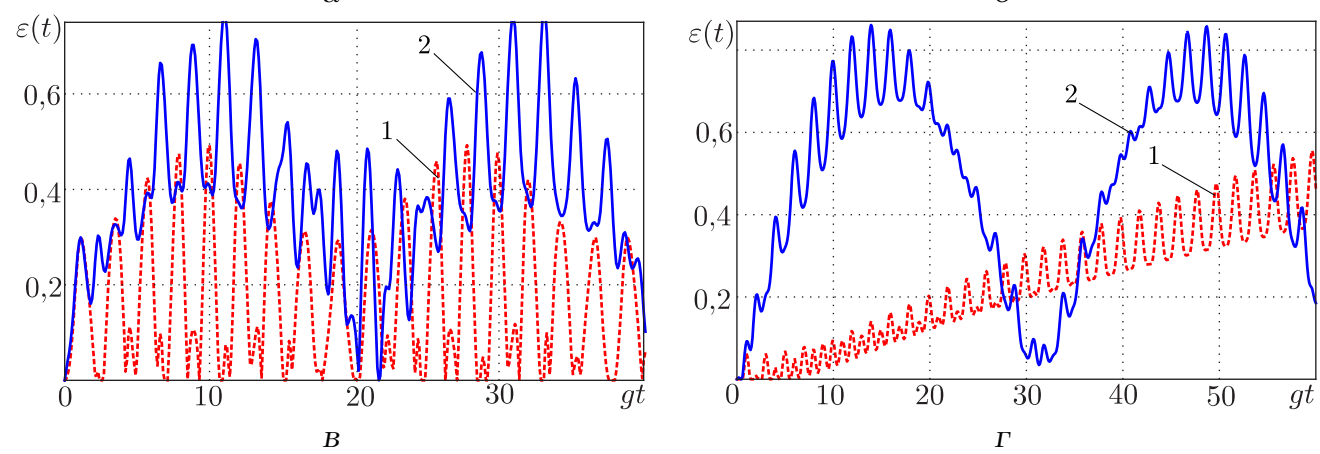

Рис. 2. Временная зависимость параметра перепутывания для параметров модели: а) $\bar{n}_{1}=$ $=\bar{n}_{2}=1, \alpha=0,1, \theta_{1}=\pi / 4, \theta_{2}=\pi / 4$ и $\Delta \varphi=0,5 \pi$ (линия 1$), \Delta \varphi=0,7 \pi$ (линия 2$), \Delta \varphi=\pi$ (линия 3); б, а) $\bar{n}_{1}=\bar{n}_{2}=0,01, \theta_{1}=\pi / 2, \theta_{2}=0, \varphi_{1}=\varphi_{2}=0$ и $\alpha=0$ (линия 1 ), $\alpha=0,01$ (линия 2), $\alpha=0,1$ (линия 3 ); в) $\bar{n}_{1}=\bar{n}_{2}=0,01, \theta_{1}=\pi / 4, \theta_{2}=\pi / 4, \varphi_{1}=\varphi_{2}=0$ и $\alpha=0,01$ (линия 1 ), $\alpha=0,1$ (линия 2 ); г) $\bar{n}_{1}=\bar{n}_{2}=0,01, \theta_{1}=\pi / 4, \theta_{2}=-\pi / 4, \varphi_{1}=\varphi_{2}=0$ и $\alpha=0,01$ (линия 1 ), $\alpha=0,1$ (линия 2) 
$\Gamma$, е представлены аналогичные графики для перепутывания при наличии диполь-дипольного взаимодействия с константой диполь-дипольного взаимодействия $\alpha=\Omega / g=0,1$. На рис. 2 показана зависимость степени перепутывания атомов от относительных фаз $\Delta \varphi=\varphi_{1}-\varphi_{2}$ поляризованных атомов для модели с $\bar{n}_{1}=\bar{n}_{2}=1$ и $\alpha=0,1$. Из рисунков хорошо видно, что выбор определенного начального состояния атомов (степени атомной когерентности или поляризации атомов) позволяет осуществлять эффективный контроль за степенью их перепутывания. На рис. 2, б, г показана зависимость степени перепутывания атомов от константы прямого диполь-дипольного взаимодействия для некогерентного (рис. 2, б) и различных когерентных начальных состояний атомов (рис. 2, в, г). Из рисунков хорошо видно, что для любых начальных состояний атомов наличие диполь-дипольного взаимодействия приводит к увеличению степени перепутывания атомов. Физически диполь-дипольное взаимодействие можно включить, уменьшая относительное расстояние между атомами в резонаторе. Причем расстояние между атомами в резонаторе можно легко контролировать. В настоящее время в современных магнитных ловушках Пауля охлаждённые ионы могут быть заперты на расстояниях порядка длины волны излучения. В этом случае параметр диполь-дипольного взаимодействия становится сравнимым с константой диполь-фотонного взаимодействия. В результате такие экспериментальные установки могут быть использованы для генерации значительной степени перепутывания атомов даже при наличии шума. Сравнение результатов, полученных нами для модели с невырожденными двухфотонными переходами, с соответствующими результатами для моделей с однофотонными переходами $[8,9]$ показывает, что для некоторых начальных состояний атомов в случае нелинейной модели может быть получена значительно более высокая степень атомного перепутывания.

3. Заключение. Таким образом, нами исследовано влияние атомной когерентности и диполь-дипольного взаимодействия на перепутывание двух атомов, взаимодействующих со слабым двухмодовым тепловым полем в идеальном резонаторе. При этом показано, что указанные механизмы могут использоваться для эффективного контроля за степенью перепутанности кубитов, необходимого при квантовой обработке информации.

Работа выполнена при финансовой поддержке Министерства образования и науки РФ (государственное задание 2.2459.2011).

\section{БИБЛИОГРАФИЧЕСКИЙ СПИСОК}

1. B. Schumacker, M. D. Westmoreland, Quantum Processes, Systems, and Information. Cambridge: Cambridge University Press, 2010. xii+469 pp.

2. M. S. Kim, J, Lee, D. Ahn, P. L. Knight, "Entanglement induced by a single-mode heat environment" // Phys. Rev. A, 2002. Vol.65, no.4, 040101(R). 4 pp., arXiv: quant$\mathrm{ph} / 0109052$.

3. L. Zhou, H. S. Song, C. Li, "Entanglement induced by a single-mode thermal field and the criteria for entanglement" // J. Opt. B: Quantum Semiclass. Opt., 2002. Vol.4, no. 6. Pp. 425-429.

4. E. K. Bashkirov, "Entanglement induced by the two-mode thermal noise" // Laser Phys. Lett., 2006. Vol.3, no. 3. Pp. 145-150, arXiv: quant-ph/0508188.

5. E. K. Башкиров, М. П. Ступачкая, "Перепутывание двух атомов, взаимодействующих с тепловым электромагнитным полем" // Компъютерная оптика, 2011. Т. 35, 
№2. C. 243-249. [E. K. Bashkirov, M. P. Stupatskaya, "Entanglement of two atoms interacting with a thermal electromagnetic field" // Komp'yuternaya optika, 2011. Vol. 35, no. 2. Pp. 243-249].

6. L. S. Aguiar, P. P. Munhoz, A. Vidiella-Barranco, J.A. Roversi, "The entanglement of two dipole dipole coupled atoms in a cavity interacting with a thermal field" // J. Opt. B: Quantum Semiclass. Opt., 2005. Vol. 7, no. 12. Pp. S769-S771.

7. X.-P. Liao, M.-F. Fang, J.-W. Cai, X.-J. Zheng, "The entanglement of two dipole dipole coupled atoms interacting with a thermal field via a two-photon process" // Chinese Phys. B, 2008. Vol. 17, no. 6. Pp. 2137-2142.

8. E. K. Bashkirov, M. P. Stupatskaya, "The entanglement of two dipole-dipole coupled atoms induced by nondegenerate two-mode thermal noise" // Laser Phys., 2009. Vol. 19, no. 3. Pp. 525-530.

9. Y.-H. Hu, M.-F. Fang, C.-L. Jiang, K. Zeng, "Coherence-enhanced entanglement between two atoms at high temperature" // Chinese Phys. B, 2008. Vol. 17, no. 5. Pp. 1784-1790.

10. Y.-H. Hu, M.-F. Fang, "Coherence-enhanced entanglement induced by a two-mode thermal field" // Commun. Theor. Phys., 2010. Vol. 54, no. 3. Pp. 421-426.

11. E. K. Bashkirov, M. S. Mastuygin, The influence of dipole-dipole interaction on entanglement in nondegenerate two-photon Tavis-Cummings model with atomic coherence, 2012. 10 pp., arXiv: 1210.3685 [quant-ph]

Поступила в редакцию 13/XI/2012;

в окончательном варианте - 27/III/2013.

\section{MSC: 81V80, 94A17}

\section{ENTANGLEMENT INDUCED BY TWO-MODE THERMAL NOISE TAKING INTO ACCOUNT THE DIPOLE-DIPOLE INTERACTION AND ATOMIC COHERENCE}

\section{E. K. Bashkirov, E. G. Mangulova}

Samara State University,

1, Academician Pavlov st., Samara, 443011, Russia.

E-mails: bash@samsu.ru, mangulova-eg@mail.ru

The entanglement of two two-level atoms with the nondegenerate two-photon transitions interacting with two-mode thermal field in lossless cavity has been considered. The possibility of entanglement degree growth in the presence of an atomic coherence and direct dipole-dipole interaction of atoms has been derived.

Key words: atomic entanglement, two-mode thermal field, dipole-dipole interaction, atomic coherence.

Original article submitted 13/XI/2012;

revision submitted $27 / \mathrm{III} / 2013$.

Eugene K. Bashkirov (Dr. Sci. (Phys. \& Math.)), Professor, Dept. of General and Theoretical Physics. Ekaterina G. Mangulova, Student, Dept. of General and Theoretical Physics. 